\title{
Semantic priming in the name retrieval of objects and famous faces
}

\author{
Markus F. Damian ${ }^{1 *}$ and Rasha Abdel Rahman ${ }^{2}$ \\ 'University of Bristol, UK \\ ${ }^{2}$ Max Planck Institute for Psycholinguistics, Nijmegen, The Netherlands
}

\begin{abstract}
Researchers interested in face processing have recently debated whether access to the name of a known person occurs in parallel with retrieval of semantic-biographical codes, rather than in a sequential fashion. Recently, Schweinberger, Burton, and Kelly (200I) took a failure to obtain a semantic context effect in a manual syllable judgment task on names of famous faces as support for this position. In two experiments, we compared the effects of visually presented categorically related prime words with either objects (e.g. prime: animal; target: dog) or faces of celebrities (e.g. prime: actor; target: Bruce Willis) as targets. Targets were either manually categorized with regard to the number of syllables (as in Schweinberger et al.), or they were overtly named. For neither objects nor faces was semantic priming obtained in syllable decisions; crucially, however, priming was obtained when objects and faces were overtly named. These results suggest that both face and object naming are susceptible to semantic context effects.
\end{abstract}

The way in which conceptual and name codes of familiar faces are accessed from perceptual input is a matter of considerable current debate in cognitive research. A comprehensive model of face recognition was proposed by Bruce and Young (1986), which assumes that access to face names is the last step in a serially arranged sequence of processing stages. According to this model, perception of a familiar face activates structural and view-independent long-term representations (face recognition units; FRUs). These FRUs are linked to amodal person identity nodes (PINs) which contain semantic-biographical information, such as occupation, hobbies, date of birth, etc. In a final step, name nodes are accessed from their corresponding PINs. Crucially, this framework assumes that face naming is conceptually mediated: there is no direct link between the recognition of a face, and the retrieval of its corresponding name.

Recently, the notion that access to face names is the final step in a series of

* Requests for reprints should be addressed to Markus F. Damian, University of Bristol, Department of Experimental Psychology, 8 Woodland Road, Bristol, BS8 ITN, UK (e-mail:m.damian@bristol.ac.uk). 
processing stages has been challenged; alternative architectures instead adapt central assumptions from McClelland and Rumelhart's (1981) interactive activation and competition (IAC) framework. For instance, in Burton and Bruce's (1992) model, PINs are activated from their corresponding FRUs, but these PINs merely serve as modality-free interfaces between FRUs on the one hand, and both semantic-biographical information and names on the other hand. It is assumed that name representations are either stored alongside semantic codes (Burton \& Bruce, 1992), or in a module separate from semantic representations (Bredart, Valentine, Calder, \& Gassi, 1995). Importantly, however, rather than viewing name retrieval as a stage subsequent to the access of semantic information, this framework postulates parallel access to semantic and name codes. Hence, the assumption of conceptual mediation prevalent in serial models of face naming is abandoned.

Evidence that could distinguish empirically between these two accounts is scant to date-both frameworks can account for basic empirical findings such that the retrieval of names is slower and more error-prone than that of object labels (see, e.g. Hanley \& Cowell, 1988; Johnston \& Bruce, 1990; Young, Ellis, \& Flude, 1988, Young, Hay, \& Ellis, 1985; Young, McWeeny, Ellis, \& Hay, 1986). A possible strategy to this aim is to investigate whether face name retrieval is subject to semantic context effects. If, as the serial account assumes, face naming mandatorily proceeds from face recognition to name retrieval via semantic representations, semantically related primes should induce priming effects. The parallel account, on the other hand, does not necessarily predict semantic effects in face naming, as names can be accessed independently of semantic codes. The absence or presence of semantic context effects could thus be used to adjudicate between the competing models. This idea was recently developed by Schweinberger, Burton, and Kelly (2001) who employed a task in which participants were presented with faces of famous people, and were asked to manually judge whether the person's first name consisted of one or two syllables ('HUGH Grant' vs. 'KEVIN Costner'). This task is assumed to require the internal generation of a phonological score of the person's name and hence is thought to exhibit similar characteristics to overt naming. It was shown that prime words that were visually presented prior to face onset and that provided partial semantic information (i.e. indicating the person's nationality, occupation, or whether or not he/she was alive or dead) had no effect on latencies. In contrast, two types of phonologically related primes that either provided partial information about the name (K. C. for Kevin Costner), or fragments of the name ('E_I__O_T_ER'), significantly facilitated the syllable judgment. A second experiment that used a semantic, rather than a name, categorization task (i.e. judging a person's nationality: British vs. American) demonstrated a semantic facilitation effect, indicating that the semantically related primes used in the first experiment were in principle able to induce semantic priming. The absence of semantic priming in the name judgment task, together with an effect of phonological relatedness, was interpreted as evidence against conceptual mediation of face name retrieval, and hence supported a parallel account.

These findings are informative but, given previous conflicting evidence regarding whether face naming is subject to semantic priming (e.g. Barry, Johnston, \& Scanlan, 1998; Brennen \& Bruce, 1991; Carson \& Burton, 2001; Young, Flude, Hellawell, \& Ellis, 1994), the reported null finding should be cautiously regarded. More specifically, Schweinberger et al. (2001) used a novel task, syllable judgment, rather than an overt naming task. This was motivated by an attempt to equate demands between a task involving name retrieval and one requiring semantic access. Underlying this is the 
assumption that syllable judgments represent overt naming in all important aspects. This assumption is not implausible - both naming and syllable decisions should require the retrieval of phonological word forms. However, the syllable decision task has, to our knowledge, not been previously used in research on language production, and it remains possible that its demand characteristics differ from 'real' speaking in some important aspects. It could be that the task is simply not sensitive enough to reveal the semantic priming effects predicted by the serial model of face name retrieval.

The following two experiments test the assumption that the syllable judgment task renders outcomes similar to an overt naming task. To this aim, we first contrast the two tasks in object, rather than face, naming (Expt 1). Semantic priming in object naming is a well-established phenomenon (see Johnson, Paivio, \& Clark, 1996, for a review). We use correct or incorrect semantic category labels of target objects as primes, for which a few previous studies (albeit mostly in the developmental domain; e.g. Ceci, 1983; Sperber, Davies, Merrill, \& McCauley, 1982) have reported semantic effects. The second experiment then uses faces as targets and hence allows a direct comparison of object and face processing characteristics. Here, 'occupation' of a target person is used as the prime - in Schweinberger et al's (2001) study, primes of this type had yielded a trend toward semantic priming (a significant priming of 21 milliseconds ( $m s$ ) in the subjects analysis, albeit only $6 \mathrm{~ms}$ and not significant when analysed by items); hence, we attempt to maximize the chances of obtaining a semantic effect.

\section{EXPERIMENT I}

\section{Method}

\section{Participants}

A sample of 40 undergraduate students at the University of Bristol participated in this experiment for course credits. All were native English speakers and had normal or corrected-to-normal vision. Of the participants, 20 were randomly assigned to the object naming task group, the other 20 performed manual syllable decisions on the object names.

\section{Materials}

A set of 30 black-and-white line drawings of common objects was selected as target stimuli. They were from five semantic categories (animals, clothing, furniture, tools, vehicles) with six exemplars each; within each category, three labels were monosyllabic, and the other three were bisyllabic. The stimuli are listed in Appendix A. The category labels served as either related or unrelated primes; each target object was paired either with its correct category label ('related' condition), or with one drawn from one of the other four semantic categories ('unrelated' condition). For each of the four experimental blocks (see below), a different 'unrelated' prime was used.

\section{Design}

The experimental design included task (naming vs. syllable decision) as a betweensubjects variable, and relatedness (related vs. unrelated) and experimental block (blocks 1-4) as within-subjects variables. Regarding the latter factor, each of the 30 target objects was paired with both the related and the unrelated category label as a prime 
once to form a single experimental block of 60 trials, and this complete pairing was presented four times across the experiment to gain more statistical power. Items were presented in a pseudo-random fashion such that neither targets nor prime words appeared on subsequent trials; a new random sequence was generated for each participant and block.

\section{Apparatus}

Stimuli were presented from an IBM-compatible computer on a 15-inch computer monitor using DMDX 2.6, developed at the University of Arizona by J. C. Forster. Objects were presented with a size of approximately $7 \times 7 \mathrm{~cm}$ as black line drawings on white background. Prime words were presented in upper-case Courier 18-point bold font, presented in the centre of the screen. In the 'naming' task group, participants donned a Sennheiser mb40 headset with attached microphone, which was connected to the sound-card of the computer and digitally recorded response times. In the 'syllable decision' task group, participants pressed one of two keys on the computer keyboard to indicate their response; assignment of keys to responses was counterbalanced across participants. The DMDX program scheduled presentation and recorded response times on each trial.

\section{Procedure}

In the 'naming' group, participants were tested individually; in the 'syllable decision' group, participants were tested in groups of up to three. At the beginning of the experiment, participants were informed that they either had to name objects presented on the screen, or had to judge their names with regard to the number of syllables. They were then familiarized with the target objects by viewing a leaflet with the 30 pictures printed on them, with the corresponding label printed below. A practice block was then administered in which each object was presented and named or categorized once; in this block, each object was preceded by a fixation cross. In the 'naming' group, responses other than those expected were corrected; in the 'syllable decision' group, the feedback message 'correct' or 'incorrect' was provided after each trial on the screen. In a second practice block of 30 trials, each object was preceded by a category label; on a random subset, the correct category label, and on the other half, the incorrect label were shown. No feedback was provided in this and the following blocks. Then, four experimental blocks of 60 trials each were administered; within each block, each object was named or categorized once preceded by the correct category label ('related' condition), and once preceded by a wrong category label ('unrelated' condition). Short breaks were provided in between the blocks. Each testing session consisted of 240 trials and lasted approximately 30 minutes.

On each individual trial, the prime word was presented for $250 \mathrm{~ms}$, followed by a blank period of $250 \mathrm{~ms}$. Then, the target object was presented for 2,000 ms. Response times were measured relative to the onset of the target; if no response occurred within $2,000 \mathrm{~ms}$, the experiment proceeded to the next trial. In the 'naming' group, the experimenter judged each response to be either correct or incorrect (which included responses other than those expected, repairs, stuttering or mouth clicks, and malfunctioning of the voice key). Each trial was followed by a 1,500 ms inter-trial interval. 


\section{Results}

Response times longer than 1,500 ms or shorter than $200 \mathrm{~ms}$, as well as trials on which participants had made an error, were excluded from the analysis. This accounted for $2.4 \%$ of the data in the naming task, and $4.8 \%$ of the data in syllable decisions. The results are displayed in Table 1 . In object naming, a priming effect of $17 \mathrm{~ms}$ was obtained that appears relatively constant across the four experimental blocks. In contrast, little or no priming is apparent in the syllable decision task.

Table I. Mean response latencies (in ms; mean error proportions in \% in parentheses) for objects (Expt I) and faces (Expt 2), varied by task (naming vs. syllable decision) and relatedness (related vs. unrelated)

\begin{tabular}{|c|c|c|c|c|c|c|c|c|}
\hline & \multicolumn{6}{|c|}{ Experimental block } & \multirow{2}{*}{\multicolumn{2}{|c|}{ Overall }} \\
\hline & \multirow[b]{2}{*}{ ects } & \multicolumn{2}{|c|}{2} & \multicolumn{2}{|c|}{3} & 4 & & \\
\hline \multicolumn{8}{|c|}{ Experiment I: Objects } & \\
\hline \multicolumn{9}{|l|}{ Naming } \\
\hline Related & $656(2.0)$ & 648 & $(1.8)$ & 646 & $(1.7)$ & $650 \quad(1.5)$ & 650 & $(1.8)$ \\
\hline Unrelated & $666(2.7)$ & 671 & $(2.3)$ & 666 & $(0.7)$ & $665 \quad(1.3)$ & 667 & (I.8) \\
\hline Effect & $10(0.7)$ & 23 & $(0.5)$ & 20 & $-1.0)$ & $15(-0.2)$ & 17 & $(0.0)$ \\
\hline \multicolumn{9}{|c|}{ Syllable Decision } \\
\hline Related & 718 (4.7) & 668 & $(3.5)$ & 622 & $(2.8)$ & $603 \quad(3.0)$ & 652 & (3.5) \\
\hline Unrelated & $727(5.5)$ & 664 & (3.7) & 630 & (3.5) & $604 \quad(2.0)$ & 656 & (3.7) \\
\hline Effect & $9(0.8)$ & -4 & $(0.2)$ & 8 & $(0.7)$ & $1(-1.0)$ & 4 & $(0.2)$ \\
\hline \multicolumn{9}{|c|}{ Experiment 2: Faces } \\
\hline \multicolumn{9}{|l|}{ Naming } \\
\hline Related & $900(5.2)$ & 852 & (3.8) & 835 & (3.5) & $831 \quad(3.8)$ & 854 & $(4.1)$ \\
\hline Unrelated & $945(6.0)$ & 887 & $(2.2)$ & 883 & $(2.0)$ & $850 \quad(1.8)$ & 891 & $(3.0)$ \\
\hline Effect & $45(0.8)$ & 35 & $(-1.6)$ & 48 & $-1.5)$ & $19(-2.0)$ & 37 & $(-1.1)$ \\
\hline \multicolumn{9}{|c|}{ Syllable Decision } \\
\hline Related & $1008(8.0)$ & 891 & $(8.7)$ & 813 & $(5.8)$ & $738 \quad(4.5)$ & 862 & $(6.8)$ \\
\hline Unrelated & 998 (8.7) & 903 & (5.8) & 806 & $(6.2)$ & $749 \quad(5.5)$ & 864 & $(6.5)$ \\
\hline Effect & $-10(0.7)$ & 12 & $(-2.9)$ & -7 & $(0.4)$ & II (I.0) & & $(-0.3)$ \\
\hline
\end{tabular}

Separate analyses of variance (ANOVAs) were performed for each level of the factor task, with relatedness and block as within-subjects variables. For the object naming task group, the effect of relatedness was significant, $F_{1}(1,19)=18.45, M S E=11,318$, $p<.001 ; F_{2}(1,29)=10.57, M S E=14,900, p<.01$. In contrast, the effect of block was not significant, $F_{1}=0.18 ; F_{2}=0.58$, and neither was the interaction between relatedness and block, $F_{1}=0.54 ; F_{2}=0.80$. In contrast, in the syllable decision task group, no significant effect of relatedness was found, $F_{1}=0.49 ; F_{2}=0.70$, but the effect of block was significant, $F_{1}(3,57)=48.86, M S E=108,351, p<.001 ; F_{2}(3,87)=109.70$, $M S E=163,866, p<.001$. The interaction between relatedness and block was not significant, $F_{1}=0.62 ; F_{2}=0.85$. In summary, relatedness mattered only in object naming, but not in syllable decision, and experimental block mattered only in syllable decision, but not in object naming. 


\section{Markus F. Damian and Rasha Abdel Rahman}

Similar ANOVAs were performed on the error percentages for each level of the factor task, with relatedness and block as within-subjects variables. For the object naming group, neither the factor relatedness was significant, $F=0.002$, nor the factor block, $F=1.79$, nor the interaction between them, $F=0.80$. For the syllable decision group, relatedness was not significant, $F=0.12$, but block was, $F(3,57)=4.68, M S E=48$, $p<.01$. The interaction between relatedness and block was not significant, $F=0.72$.

\section{Discussion}

A significant semantic priming effect was obtained only in the overt object naming task but not in the syllable classification task. Both tasks showed overall comparable response times, but whereas in naming, response times remained roughly at the same level across the experimental blocks, a considerable practice effect was obtained in syllable decisions. In neither group did the variable of block interact with the relatedness factor; hence, the repeated presentation of a relatively small set of targets did not affect the findings.

The finding that the naming of an object is executed faster when it is preceded by the correct categorical label than when preceded by the label of a different semantic category is expected from previous studies on this issue. On the other hand, the absence of a parallel semantic priming effect in the syllable decision task performed on the object label is rather surprising. Given that both tasks appear to require the generation of the target's phonological score, they could be expected to be approximately equally sensitive to semantic constraints. Contrary to these predictions, the results suggest a clear task difference in this regard. Crucially, the findings point out that a failure to obtain semantic effects in the syllable judgment task does not automatically imply a corresponding failure in an overt naming task.

The following experiment is identical in all important aspects to Expt 1, except that instead of common objects, the faces of familiar people are used as targets, and instead of semantic category labels, the corresponding occupational labels are used as prime words. Adapted from Schweinberger et al. (2001), the syllable decision is performed on the person's first name; this condition hence provides a partial replication of Schweinberger et al's study. According to Schweinberger et al., face processing is not subject to semantic effects and, hence, an absence of semantic priming is predicted for both overt naming and syllable judgments. In contrast, the results of our first experiment suggest that, at least in object processing, the two tasks differ in important ways, with only object naming exhibiting semantic effects. The crucial point of interest in Expt 2 is hence the overt naming condition. Here, a failure to obtain semantic priming would provide evidence for a parallel account of face name retrieval.

\section{EXPERIMENT 2}

\section{Methods}

\section{Participants}

A sample of 40 undergraduate students at the University of Bristol, none of whom had participated in Expt 1, took part in this experiment for course credits. All were native English speakers and had normal or corrected-to-normal vision. A further five participants (three in the 'naming', and two in the 'syllable decision' group) were excluded 
from the experiment because in the initial familiarization phase (see below), they indicated that they were not fully familiar with all the faces used as targets.

\section{Materials}

A set of 30 images displaying faces of celebrities were selected as targets. These stemmed from five occupational categories (actors, athletes, politicians, singers, TV personalities), with three people in each category having monosyllabic first names, and the other three having bisyllabic first names. The stimuli are listed in Appendix B. Occupational labels served as related or unrelated primes; each face was paired either with the correct occupational category label ('related' condition), or with one drawn from one of the other four categories ('unrelated' condition). For each of the four experimental blocks, a different 'unrelated' prime was used.

\section{Design}

The design was identical to that used in Expt 1, with task (naming vs. syllable decision) as a between-subjects variable, and relatedness (related vs. unrelated) and block (1-4) as within-subjects variables. Again, items were presented in a pseudo-random fashion such that neither targets nor prime words appeared on subsequent trials; a new random sequence was generated for each participant and block.

\section{Apparatus}

This was the same as in Expt 1. The images were edited so as to delete all information apart from the face, standardized to a size of approximately $7 \times 7 \mathrm{~cm}$, and displayed in greyscale on a white background. Prime words were presented in upper-case Courier 18-point bold font, presented in the centre of the screen.

\section{Procedure}

The procedure followed that used in Expt 1 in all important respects. Again, in the 'naming' group, participants were tested individually, whereas in the 'syllable decision' group, participants were tested in groups of up to three. Participants were familiarized with the faces by providing them with a leaflet that contained each face with the correct name printed below, and were then instructed with regard to their task. Then, two practice blocks were administered; in the first, each target was preceded by a fixation cross, and feedback with regard to the correctness of the response was provided. In the second practice block, a random subset of the targets were preceded by the correct occupational label, and the remaining targets were preceded by a label other than the correct one. Again, four experimental blocks of 60 trials each were conducted, with short breaks in between them.

Each individual trial had the same temporal structure as in Expt 1: the prime word was presented for $250 \mathrm{~ms}$, followed by a blank period of $250 \mathrm{~ms}$. Then the target appeared for $2,000 \mathrm{~ms}$, and responses were measured relative to its onset. If no response occurred within that period, the experiment proceeded to the next item. Each trial was followed by a $1,500 \mathrm{~ms}$ inter-trial interval.

\section{Results}

Response times longer than $1,800 \mathrm{~ms}$ or shorter than $250 \mathrm{~ms}$, as well as trials on which participants had made an error, were excluded from the analysis, accounting for $7.5 \%$ of 
the data in naming, and $10.2 \%$ in syllable decisions. The results are displayed in the bottom half of Table 1. Replicating the results reported by Schweinberger et al. (2001), little or no priming was obtained in the syllable decision task. Crucially, however, substantial priming emerged in the naming of faces.

Separate ANOVAs were performed for each level of the factor task, with the variables relatedness and block as within-subjects factors. For the naming task, a significant effect of relatedness was found, $F_{1}(1,19)=42.15, M S E=53,141, p<.001$; $F_{2}(1,29)=47.72, M S E=79,607, p<.001$. Also, the main effect of block was significant, $F_{1}(3,57)=18.84, M S E=49,740, p<.001 ; F_{2}(3,87)=55.57, M S E=79,943, p<.001$, but the relatedness $\times$ block interaction was not, $F_{1}=1.59 ; F_{2}=1.53$. In the syllable decision task, the effect of relatedness was not significant, $F_{1}=0.05 ; F_{2}=0.01$, but the main effect of block was, $F_{1}(3,57)=65.61, M S E=504,783, p<.001 ; F_{2}(3,87)=281.39$, $M S E=717,688, p<.001$. The interaction between relatedness and block was not significant, $F_{1}=0.72 ; F_{2}=0.75$. In summary, relatedness mattered only in face naming, but not in the syllable decision task. The factor experimental block mattered in both tasks, but the block factor did not interact with relatedness in either group.

Similar ANOVAs were performed on the error percentages for each level of the factor task, with relatedness and block as within-subjects variables. For the object naming group, the factor relatedness was not significant, $F=2.89$, but the factor block was significant, $F(3,57)=5.76, M S E=75, p<.01$. The interaction between block and relatedness was not significant, $F=1.52$. For the syllable decision group, the factor relatedness was not significant, $F=0.13$, but the factor block was, $F(3,57)=6.17$, $M S E=85, p<.01$. The interaction between relatedness and block was not significant, $F=2.10$.

\section{Discussion}

Experiment 2 replicated Schweinberger et al's (2001) observation that semantically related primes fail to yield a relatedness effect when faces had to be manually categorized according to the number of syllables of the corresponding names. Crucially, however, a robust relatedness effect was obtained when faces had to be named, rather than categorized. These findings imply that, at least as far as these experimental paradigms are concerned, there are no important differences between objects and faces; rather, the presence or absence of a semantic effect depends on the task that is used. Clearly, the fact that syllable decisions on face names failed to show semantic priming should not be taken as evidence against conceptual mediation in face naming, as suggested by Schweinberger et al.

\section{GENERAL DISCUSSION}

This study compared directly the effects of a categorical prime on object and face name retrieval, assessed either by an overt naming task, or by a syllable decision performed on the object label or the person's first name. The data showed parallel findings for both types of targets; namely, robust semantic priming in overt naming, but no such effect in the syllable decision task. These findings suggest that the processing of faces and objects appears to be rather similar, but that the two tasks differ in important aspects.

The presence or absence of semantic context effects in face processing is relevant 
regarding the issue of whether access to people's names occurs serially, or in parallel, to semantic-biographical information: Schweinberger et al. (2001) had taken their failure to obtain semantic priming with a syllable judgment task as evidence for parallel access to semantics and names, and against a serial account. The current findings suggest that the syllable judgment task is insensitive to semantic priming not only in face, but also in object naming; hence Schweinberger et al's null finding should not be taken as evidence against conceptual mediation in the name retrieval of faces.

On the other hand, our positive finding of semantic priming in both object and face naming does not necessarily suggest that face naming (or object naming, for that matter) is conceptually mediated; semantic effects are potentially compatible with both serial and parallel models of face naming. For instance, in the related domain of visual word recognition, virtually all accounts proposed to date (e.g. Coltheart, Rastle, Perry, Langdon, \& Ziegler, 2001; Plaut, McClelland, Seidenberg, \& Patterson, 1996; Seidenberg \& McClelland, 1989) agree on the assumption that word naming can be achieved subsemantically. However, the fact that semantic context effects are found in word naming (see Neely, 1991, for a review) is not generally considered as being at odds with a subsemantic route to word naming. Hence, using the presence and absence of such semantic effects to argue for or against conceptual mediation in a particular domain is problematic: a positive finding (as in the naming tasks presented here) is theoretically uninformative, whereas a negative finding (such as in Schweinberger et al., 2001) exhibits all the known problems associated with null findings, as our experiments demonstrate. We would argue that alternative paradigms and techniques, such as those used in Abdel Rahman, Sommer, and Schweinberger (2002), or neuropsychological investigations (e.g. Brennen, 1999; Hodges \& Greene, 1998), might be better suited to advance this issue.

If theoretical conclusions about naming retrieval are based on the presence or absence of semantic effects, then the naming task is probably preferable, because it requires a genuine speech response. On the other hand, manual name classification tasks of various kinds (e.g. van Tourennout, Hagoort, \& Brown, 1997; Wheeldon \& Levelt, 1995) have been shown to be sensitive to various aspects of phonological encoding, such as its time course. Furthermore, in many experimental situations, in particular electrophysiological or neuroimaging studies of language production, manual classification tasks are preferable to overt verbal responses for methodological reasons such as the avoidance of motion artifacts. In other circumstances (such as reported by Schweinberger et al., 2001), a manual task such as a syllable decision judgment might be chosen to equate task demands with a further task, such as semantic categorization. Hence, for future research, it would be important to know in what aspects manual tasks differ from overt naming.

Unfortunately, it remains unresolved exactly why the syllable judgment task does not exhibit semantic context effects. One possible reason for the failure to obtain semantic effects is that in our procedure, the prime word itself provides a syllable structure which matches or mismatches that of the target. If participants inadvertently categorized the prime words with regard to their syllable structure, this might create enough noise to prevent detection of a semantic relatedness effect. This possibility was investigated in the following way: out of the five category labels used in the first experiment, one ('tool') is monosyllabic, and one ('clothing') is bisyllabic. A subset of the trials from this experiment were reclassified into those in which the prime and target matched in their numbers of syllables (e.g. monosyllabic targets preceded by 'tool', and bisyllabic targets preceded by 'clothing'), and those in which they did not match (vice versa). The results 
showed an average response latency of $648 \mathrm{~ms}$ for congruent trials, and one of $646 \mathrm{~ms}$ for incongruent trials. These findings do not provide evidence that prime syllable structure might have interfered with the task of categorizing target syllable structure. Hence, the reason why syllable decisions render results that differ from overt speaking regarding semantic context effects remains unexplained. Nevertheless, the findings reported here certainly warrant caution with regard to substituting one task for another without first comparing them on the variable of main interest.

\section{Acknowledgement}

We would like to thank Rebecca Thompson for providing us with familiarity norms of a subset of the face images used in this study.

\section{References}

Abdel Rahman, R., Sommer, W., \& Schweinberger, S. R. (2002). Brain-potential evidence for the time course of access to biographical facts and names of familiar persons. Journal of Experimental Psychology: Learning, Memory, and Cognition, 28, 366-373.

Barry, C., Johnston, R. A., \& Scanlan, L. C. (1998). Are faces 'special' objects? Associative and semantic priming of face and object recognition and naming. Quarterly Journal of Experimental Psychology, 51A, 853-882.

Bredart, S., Valentine, T., Calder, A., \& Gassi, L. (1995). An interactive activation model of face naming. Quarterly Journal of Experimental Psychology, 48A, 466-486.

Brennen, T. (1999). Face naming in dementia: A reply to Hodges and Green (1998). Quarterly Journal of Experimental Psychology, 52A, 535-541.

Brennen, T., \& Bruce, V. (1991). Context effects in the processing of familiar faces. Psychological Research, 53, 296-304.

Bruce, V., \& Young, A. (1986). Understanding face recognition. British Journal of Psychology, 77, $305-327$.

Burton, A. M., \& Bruce, V. (1992). I recognize your face but I can't remember your name: A simple explanation? British Journal of Psychology, 83, 45-60.

Carson, D. R., \& Burton, A. M. (2001). Semantic priming of person recognition: Categorical priming may be a weaker form of the associative priming effect. Quarterly Journal of Experimental Psychology, 54A, 1155-1179.

Ceci, S. J. (1983). Automatic and purposive semantic processing characteristics of normal and languge/learning-disabled children. Developmental Psychology, 19, 427-439.

Coltheart, M., Rastle, K., Perry, C., Langdon, R., \& Ziegler, J. (2001). DRC: A dual route cascaded model of visual word recognition and reading aloud. Psychological Review, 108, 204-256.

Hanley, J. R., \& Cowell, E. S. (1988). The effects of different types of retrieval cues on the recall of names of famous faces. Memory and Cognition, 16, 545-555.

Hodges, J. R., \& Greene, J. D. W. (1998). Knowing about people and naming them: Can Alzheimer's disease patients do one without the other? Quarterly Journal of Experimental Psychology, 51A, 121-134.

Johnson, C. J., Paivio, A., \& Clark, J. M. (1996). Cognitive components of picture naming. Psychological Bulletin, 120, 113-139.

Johnston, R. A., \& Bruce, V. (1990). Lost properties? Retrieval differences between name codes and semantic codes for familiar people. Psychological Research, 52, 62-67.

McClelland, J. L., \& Rumelhart, D. E. (1981). An interactive activation model of context effects in letter perception. Part 1: An account of basic findings. Psychological Review, 88, 375-407.

Neely, J. H. (1991). Semantic priming effects in visual word recognition: A selective review of 
current findings and theories. In D. Besner \& G. W. Humphreys (Eds.), Basic processes in reading: Visual word recognition (pp. 264-336). Hillsdale, NJ: Erlbaum.

Plaut, D. C., McClelland, J. L., Seidenberg, M., \& Patterson, K. (1996). Understanding normal and impaired word reading: Computational principles in quasi-regular domains. Psychological Review, 103, 56-115.

Schweinberger, S. R., Burton, A. M., \& Kelly, S. W. (2001). Priming the access to names of famous faces. Journal of British Psychology, 92, 303-317.

Seidenberg, M. S., \& McClelland, J. L. (1989). A distributed developmental model of word recognition. Psychological Review, 96, 523-568.

Sperber, R. D., Davies, D., Merrill, E. C., \& McCauley, C. (1982). Cross-category differences in the processing of subordinate-superordinate relationships. Child Development, 53, 1249-1253.

van Tourennout, M., Hagoort, P., \& Brown, C. M. (1997). Electrophysiological evidence on the time course of semantic and phonological processes in speech production. Journal of Experimental Psychology: Learning, Memory, and Cognition, 23, 787-806.

Wheeldon, L. R., \& Levelt, W. J. M. (1995). Monitoring the time course of phonological encoding Journal of Memory and Language, 34, 311-334.

Young, A. W., Ellis, A. W., \& Flude, B. M. (1988). Accessing stored information about familiar people. Psychological Research, 50, 111-155.

Young, A. W., Flude, B. M., Hellawell, D., \& Ellis, A. W. (1994). The nature of semantic priming effects in the recognition of familiar people. British Journal of Psychology, 85, 393-411.

Young, A. W., Hay, D. C., \& Ellis, A. W. (1985). The faces that launched a thousand slips: Everyday difficulties and errors in recognizing people. British Journal of Psychology, 76, 495-523.

Young, A. W., McWeeny, K. H., Ellis, A. W., \& Hay, D. C. (1986). Naming and categorizing faces and written names. Quarterly Journal of Experimental Psychology, 38, 297-318.

Received 2 August 2002; revised version received 6 February 2003

\section{Appendix A}

\section{Stimuli used in Experiment I}

ANIMALS: fish, mouse, snake, camel, spider, zebra CLOTHING: dress, hat, sock, mitten, sandal, trousers FURNITURE: bed, chair, desk, sofa, table, wardrobe TOOLS: comb, drill, saw, hammer, scissors, spanner

VEHICLES: car, ship, train, lorry, moped, tractor

\section{Appendix B}

\section{Stimuli used in Experiment 2}

ACTORS: Bruce Willis, Jack Nicholson, Sean Connery, Arnold Schwarzenegger, Dustin Hoffman, Kevin Costner

ATHLETES: Frank Bruno, Pete Sampras, Tim Henman, Andre Agassi, David Beckham, Michael Owen

POLITICIANS: Bill Clinton, George W. Bush, John F. Kennedy, Boris Yeltsin, Michael Heseltine, Tony Blair

SINGERS: Bob Geldof, Mick Jagger, Phil Collins, Elton John, Elvis Presley, Robbie Williams

TV PERSONALITIES: Ben Elton, Keith Chegwin, Paul Daniels, Michael Parkinson, Philip Schofield, Tony Hart 